\title{
Contribución de las competencias emocionales a mejorar la calidad del rendimiento académico
}

\author{
Damaris Rodríguez Peralta ${ }^{1}$
}

\section{RESUMEN}

El presente artículo presenta la revisión y recopilación teórica sobre aspectos vinculados al tema rendimiento académico y competencias emocionales; entre las que se mencionan aspectos relacionados a las emociones, la inteligencia emocional, educación emocional; así como también algunos hallazgos de investigaciones encontrados en la literatura sobre el tema. Para ello, se apoya en algunos estudios realizados a nivel internacional sobre el tema en cuestión. El rendimiento académico se vincula a los resultados de notas y promedios obtenidos por los estudiantes como resultado de ser aprobado o reprobado en sus materias, tomando estos indicadores como un elemento objetivo a tomar en cuenta en el desempeño académico de los estudiantes universitarios, sin tomar en cuenta otros indicadores de carácter subjetivos y sociales como parte de esos resultados cuantitativos. Las competencias emocionales y la inteligencia cognitiva, deben formar parte del currículo académico, por su importante contribución al desarrollo integral del estudiante.

Palabras clave: calidad, rendimiento académico, competencias emocionales, inteligencia emocional, educación emocional.

Recibido: 06 de octubre de 2017

Aceptado: 30 de noveimbre de 2017

1 Docente UNAN-Managua FAREM-Estelí. Estudiante del Doctorado Gestión de la Calidad de la Investigación. UNAN-Managua FAREM-Estelí. Correo electrónico: dacareduar20@yahoo.es 


\title{
Contribution of emotional competences to improve the quality of academic performance
}

\begin{abstract}
This article presents theoretical review and compilation on aspects related to academic performance and emotional competences; it mention aspects related to emotions, such as; emotional intelligence, emotional education; As well as some research findings found in the literature on the subject. This is supported by some studies carried out at the international level on the subject. Academic achievement is linked to the results of grades and averages obtained by students as a result of passing or failing in their subjects, taking into account these indicators as an objective element in the academic performance of university students, without taking into account of other subjective and social indicators as part of these quantitative results. Emotional competencies and cognitive intelligence must form part of the academic curriculum, for its important contribution to the integral development of the student.
\end{abstract}

Keywords: quality, academic performance, emotional competences, emotional intelligence, emotional education. 


\section{INTRODUCCIÓN}

La educación superior en América Latina experimentó, en la década de 1990, un marcado interés por la calidad educativa, al reconocer en ella la principal herramienta para responder a las exigencias y demandas educativas en un contexto marcado por desafíos propios del proceso de la globalización. En el marco de la globalización, la educación superior en América Latina enfrenta la necesidad de una convergencia y articulación de sus sistemas, así como la construcción y consolidación del espacio latinoamericano de educación superior, que son importantes desafíos ante la fragmentación, diversificación institucional y disparidad que se presenta en la educación superior desde la década de 1990| (Garbanzo Vargas, 2007).

La calidad educativa es uno de los términos más utilizados en el ámbito educativo, constantemente escuchamos hablar que necesitamos mejorar en este campo, desde nuestro contexto universitario se realizan distintas acciones a nivel de plan de estudios, programas, formación profesional, capacitaciones etc. Pero aun la Educación Superior en Nicaragua tiene grandes retos, que conllevan a realizar transformaciones en los procesos educativos, que den respuestas a las necesidades de una sociedad más competitiva; es por ello que las universidades deben estar preparadas para enfrentar estos retos que vienen con los avances, científicos, tecnológicos, políticos y culturales del mundo moderno para satisfacer las necesidades educativas actuales.

De acuerdo a los resultados del estudio realizado por Gaeta y López, sobre la relación que guardan las competencia emocionales y rendimiento académico en estudiantes universitarios mexicanos, se utilizaron el Trait Meta-Mood Scale(TMMS-24), la Escala de Habilidades Sociales (EHS) y el promedio académico final de los estudiantes... encontrando que existe una coorrelación significativa negativa entre el rendimiento académico y las interacciones sociales. Se enfatiza la relevancia de desarrollar competencias emocionales en los estudiantes, que contribuyan a su bienestar y equilibrio emocional ( Gaeta González \& López Gárcia, 2013, pág. 20).

El presente artículo está dirigido a explorar algunos trabajos realizados en función de la calidad del rendimiento académico y competencias emocionales en estudiantes Universitarios el cual en su estructura intenta dar respuesta a las siguientes interrogantes: ¿Cómo se ha venido trabajando el tema de calidad educativa en el ámbito universitario?, ¿Qué experiencias existen sobre el rendimiento académico y las competencias emocionales en estudiantes universitarios?, ¿Cuál ha sido la metodología que se ha utilizado?, ¿Cuáles han sido los aportes a la ciencia?, ¿Cuál es el rol de las competencias emocionales en la educación superior?, ¿Qué es lo nuevo por aportar respecto al tema?

\section{Calidad Educativa}

La calidad educativa es un concepto amplio que en la actualidad es un tema de debate que tiene que ver con voluntad política, presupuesto y otros procesos de carácter objetivos y subjetivos, no solo tienen que ver con conocimientos teóricos, sino en valores, ética, afectividad, cambio de actitud que conlleven al/la estudiante a prepararse para la vida. Es decir, la calidad posee múltiple dimensiones, visiones $\mathrm{e}$ interpretaciones. Pero el problema no consiste en buscar una nueva definición de calidad; pues ya existen muchas en la literatura actual, sino determinara aquella que más convenga a la evaluación en las condiciones de la realidad latinoamericana, sin olvidar que la calidad tienen que estar conjugada con la pertinencia y el impacto, pues no se puede concebir una institución universitaria de calidad que no sea pertinente en su entorno social (Agulla Cabrera, 2005).

De acuerdo a los principios de Freire los cuales se basan en una concepción humanista, muestran un absoluto respeto por el ser humano al igual que nuestro modelo educativo de la UNAN Managua en el que plantea que el proceso de enseñanza-aprendizaje 
tiene como base un modelo pedagógico que centra su atención en el estudiante que asume un rol activo y participativo con una alta responsabilidad en el desarrollo de un aprendizaje autónomo y estratégico. El propósito fundamental es promover la construcción de saberes que tengan significado y relevancia en la solución de problemas reales y cotidianos. En la formación científica y humanística de los estudiantes se propicia la interacción y la autorreflexión, prestando así atención al desarrollo del pensamiento analítico y crítico. Esto a su vez, contribuye a la formación integral de un profesional competente para desenvolverse e integrarse con éxito en el ámbito profesional y social (UNAN, 2011).

Este planteamiento conlleva a un gran reto de asumir con responsabilidad desde la docencia, de tal forma que los y las docentes sean facilitadores/as de estos procesos en los y las discentes, esto me lleva a cuestionarme. ¿Estamos realmente los/as docentes consiguiendo que nuestros estudiantes sean críticos autocríticos, analíticos, empáticos, con responsabilidad social, reflexivos, asertivos etc., para contribuir con su calidad como profesionales, o hace falta trabajar en ello?

Desde el punto de vista Humanista de Maslow cuando explica la motivación del ser humano al plantearse constantemente metas hasta conseguir la autorrealización como una necesidad humana. Maslow argumenta que las necesidades superiores son tan reales y tan esenciales a la condición humanan como la necesidad de comer (Maslow, 1991). Del mismo modo también lo plantea el modelo Educativo de la UNAN Managua en su misión de Formar profesionales y técnicos integrales desde y con una concepción científica y humanista del mundo, capaces de interpretar los fenómenos sociales y naturales con un sentido crítico, reflexivo y propositivo, para que contribuyan al desarrollo social.... (UNAN, 2011).

Sin duda alguna la calidad educativa es la preocupación más sentida y también el logro más trabajado en las reformas educativas en Centroamérica. La calidad según la perspectiva de una inversión de capital humano que debe producir rendimientos mensurables, necesariamente converge en los rendimientos académicos trabajados en los procesos educativos con todos los elementos y medios que lo dinamizan. La calidad se empalma, de esta manera, con eficiencia interna del sistema educativo, por cuanto el resultado global de su acción son los resultados del aprendizaje del educando, planificados y organizados de antemano, para ser medidos con los estándares académicos establecidos.

\section{Contexto de la situación Educativa en Nicaragua}

la constitución política de Nicaragua en su Título VII, capitulo único, arto.16 expresa que la educación tienen como objetivo la formación plena e integral del Nicaragüense; dotarlo de una conciencia crítica, científica y humanista; desarrollar su personalidad y el sentido de su dignidad; y capacitarlo para asumir las tareas de interés común que demanda el progreso de la nación; por consiguiente la educación es un factor fundamental para la trasformación y el desarrollo del individuo y la sociedad (Nicaragua, 2008). La educación es un derecho inherente al ser humano lo que posibilita su bienestar personal, económico político y social en beneficio de cada uno de los contextos en los que se desarrolle. En el año 1981 el gobierno Sandinista declaró que la educación es un proceso continuo, recurrente y permanentede formación ydesarrollo delapersonalidad; en el año 1983 la Educación se concibe como un sistema integrado por varios subsistemas y es en el año 1984 para concretar esos principios el sistema Educativo Nacional queda constituido por cinco subsistemas como es el sistema de Educación General, Básica y Media, subsistema de Educación Popular Básica, Educación Técnica, Educación Superior y Capacitación (Bautista Arríen, Xabier Gorostiaga, \& Tunnermann Bernheim, 1997, pág. 22).

A partir de 1990, en un nuevo contexto político por el cambio de gobierno, el Ministerio de Educación organizó la educación con una visión completamente 
opuesta a las políticas educativas, estructuras y valores construidas por el gobierno sandinista, viéndose disminuida la planificación educativa a funciones tecnocráticas y mecanismos burocráticos, poniéndose en práctica la venta de servicios educativos a la población nicaragüense, y asignándose, a la educación pública, "valor de mercado"

En 1993, se estableció el modelo de Autonomía Escolar como política educativa que excluyó a la niñez y juventud del derecho a tener una educación pública gratuita y de calidad, ocasionando un retorno impactante del analfabetismo al país, carencia de un Plan Nacional de Desarrollo y la falta de participación de la sociedad civil en la formulación y gestión de las políticas educativas. En el Ministerio de Educación se generó dependencia de consultores externos, préstamos y donaciones de organismos internacionales, los que no fueron utilizados con eficiencia en función de los resultados educativos del país. De 1999 a 2001, se formuló la Estrategia Nacional de Educación y el Plan Nacional de Educación 2001-2015, definiéndose una visión de mediano y largo plazo de la educación nicaragüense. Entre el año 2001 y el año 2006, continuó el proceso de descentralización y desconcentración de funciones y recursos financieros, expresados en los modelos de autonomía escolar y municipalización. En 2003, el Ministerio de Educación Cultura y Deportes (MECD) formuló el Marco Operativo del Plan Nacional de Educación, y creó nuevas funciones y estructuras que fortalecieron la Autonomía Escolar, proyectada como un modelo de descentralización de la educación. Sin embargo, en su estudio El derecho a la educación en Nicaragua, el Dr. Miguel de Castilla, lo perfila como un modelo de desconcentración financiera del Presupuesto Nacional de la República, desde la sede central del Ministerio de Educación hacia los centros educativos" (Ministerio de Educación, 2008).

En la actualidad hablar de calidad se ha convertido en un tema coyuntural al referirse a calidad educativa, calidad de vida, calidad del agua etc., es un tema para profesionales académicos y no académicos, políticos, religiosos, investigadores etc. Se han realizado muchos esfuerzos en función de elevar la calidad de la educación. En lo que respecta a la situación actual de la educación emocional en Nicaragua, a pesar de los esfuerzos que se vienen realizando, hablar de calidad no significa simplemente explicarlo a través de un concepto sino de una situación práctica en la que se retomen tanto elementos objetivos que tienen que ver con la eficiencia, eficacia, formación, innovación etc., sino también incluir elementos subjetivos como la actitud, motivación, autoestima, el bienestar psicológico, la satisfacción académica, los estilos de aprendizajes entre otros. Como lo ostenta la teoría sistémica desde su perspectiva holística que permite integrar un conjunto de elementos que contribuyen al desarrollo humano.

En busca de la calidad la UNAN Managua en el 2002 llevó a cabo su primer proceso de autoevaluación institucional con fines de mejora en el marco del proyecto de modernización y acreditación de la educación terciaria (PMAET 2001). Entre 2013- 2014 efectúa su segundo proceso de autoevaluación con fines de mejora y de esta manera da cumplimiento a la ley 704 (ley creadora del Sistema Nacional para el Aseguramiento de la Calidad de la Educación y Reguladora del consejo Nacional de Evaluación y Acreditación), que establece que cada una de las instituciones de Educación Superior de Nicaragua realizará procesos de autoevaluación institucional que les permita identificar sus fortalezas, sus debilidades y formular planes de mejora que garanticen la calidad de la educación (UNAN, Managua, 2017, pág. 23)

\section{Rendimiento académico}

El tema rendimiento académico es ineludible para referirse al abordaje de la calidad de la educación superior, debido a que desde el punto de vista académico es un indicador de la realidad educativa de los y las estudiantes (Ibarra \& Michalus, 2010). Definen rendimiento académico como el promedio de materias aprobadas anualmente. El mundo moderno 
requiere hablar de calidad del Rendimiento Académico en el ámbito de la educación de forma amplia e integradora, tomando en cuenta tanto aspecto que están relacionados a las funciones cognitivas del ser humano como la inteligencia, el aprendizaje, la memoria y aquellos aspectos de índole afectivos emocionales y sociales que contribuyan a elevar la calidad como seres humanos y elevar el nivel como futuros profesionales.

Según Garbanzo Vargas (2007), en materia del rendimiento académico de la educación superior la mayoría de los estudios son cuantitativos, con un marcado interés en el campo económico son pocas las investigaciones que hacen un abordaje cualitativo del problema. No obstante, sus resultados han permitido identificar factores que favorecen o limitan el desempeño académico como lo señala en su estudio sobre los componentes asociados al rendimiento académico en estudiantes universitarios.

Tomando en cuenta la preocupación planteada en el modelo educativo de la UNAN Managua (2011) en el que se plantea que en nuestras facultades no existen evidencias de análisis cualitativos, ni de medidas para superar el rendimiento académico. No se investiga sobre las causas del bajo rendimiento académico ni sobre la deserción estudiantil... Nuestra casa de estudio es un escenario en el que constantemente los y las estudiantes solicitan algún servicio de atención en el cual su primera manifestación clara es la poco o ninguna valoración positiva de sí mismo, no se concentran en clase, han perdido el interés, etc. Para estudiar el tema competencias emocionales es importante conocer sobre ¿Qué son las emociones? ¿Qué es la educación emocional? ¿A que nos referimos con la inteligencia Emocional? ¿Cuándo nos referimos a las competencias Emocionales?

\section{Las Emociones}

Las emociones constituyen un componente clave que configura la vida afectiva de las personas, estableciendo en cada una diferentes capacidades y habilidades que, si se aprenden adecuadamente en los diversos ámbitos en que vive, pueden ayudarle a enfrentarse a la incertidumbre, resolver problemas y conflictos, comunicar, colaborar y trabajar con otros; es decir, ser feliz y procurar que los demás también lo sean.

La teoría más antigua que se conoce sobre emociones es la de "James-Lange". William James, a finales del siglo XIX, sugirió que las emociones consistían en la percepción de un estímulo, que se continuaba con la experiencia de la emoción y se finalizaba con la emisión de una conducta. Por la misma época, el médico Cari Lange proponía un enfoque similar, enfatizando la importancia de los eventos sensoriales en la emoción. Aunando las dos aportaciones, la integración de los procesos perceptivos y valorativos de los cambios fisiológicos compone la primera teoría que es reconocida como el antecedente de las modernas teorías centradas en los aspectos cognitivos, fisiológicos y sociales de la emoción. Según este enfoque, la emoción sería una entidad monolítica que descansaría en las dimensiones de "intensidad de la activación" y "afecto positivo vs afecto negativo". Años más tarde, en 1937, James W. Papez formula su conocido circuito neural que implica al hipotálamo y al sistema límbico como responsables del sustrato químico de las experiencias emocionales. Según su argumentación, las aferencias sensoriales componen tres rutas; una se dirige a la corteza y estaría relacionada con las cogniciones, otra hacia los ganglios básales, implicando a los movimientos, y una tercera hacia el hipotálamo, que se relacionaría con las sensaciones (Linares Ramos, 2009).

Existen tres componentes de una emoción: neurofisiológico, conductual y cognitivo. La neurofisiología se manifiesta en respuesta como: taquicardia, sudoración, vasoconstricción, hipertensión, tono muscular, rubor, resequedad en la boca, cambio en los neurotransmisores, secreciones hormonales, respiración etc. Todo esto son respuestas involuntarias, que el sujeto no puede controlar, sin embargo, se pueden prevenir con técnicas apropiadas como la relajación. La observación de la conducta en un individuo permite 
inferir que tipo de emociones está experimentando. Las expresiones faciales, el lenguaje verbal y no verbal, el tono de voz, volumen, ritmo, movimiento del cuerpo etc., aportan señales de bastante precisión sobre el estado emocional. El componente cognitivo hace que califiquemos un estado emocional y le demos un nombre. El etiquetado de las emociones está limitado por el dominio del lenguaje. Dado que la introspección a veces es el único método para llegar al conocimiento de las emociones de los demás, las limitaciones del lenguaje imponen serias restricciones a este conocimiento, al mismo tiempo dificultan el reconocimiento de las propias emociones (Bisguerra Alzina, Eduación Emocional y Competencias Básicas para la Vida, 2003).

Las investigaciones de Cannon sobre las emociones revelaron que el dolor, el hambre y las emociones fuertes como el miedo y la rabia provocan modificaciones que se caracterizan por su naturaleza refleja en cuanto reacción orgánica típica que se manifiesta gracias a un automaticismo heredado; por lo que esas modificaciones revelan un carácter racional desde el punto de vista biológico (Vigotsky, 2004).

La emoción son sensaciones que se experimentan tanto a nivel interno como externo que surgen como respuestas a estructuras significativas ante diferentes situaciones de la vida cotidiana del ser humano.

\section{Educación Emocional}

La educación emocional es una respuesta a necesidades afectivas y sociales que en nuestro contexto no se encuentran incluidas en el currículo académico entre las que se encuentran la depresión, el miedo, estrés, ansiedad, violencia, consumo de drogas, baja autoestima, falta de habilidades sociales etc. Es importante mencionar que la vida académica de los y las estudiantes no solamente gira alrededor de la capacidad intelectual del estudiante, el cual siempre se ha visto asociado al rendimiento académico sino en formar individuos felices, sanos mentalmente, con una adecuada inteligencia emocional, que se planteen retos, capaces de resolver problemas y saber negociar situaciones de la vida cotidiana.

La educación emocional se propone el desarrollo de competencias emocionales. Concebimos la educación emocional como un proceso educativo, continuo y permanente, que pretende potenciar el desarrollo de las competencias emocionales como elemento esencial del desarrollo integral de la persona, con objeto de capacitarle para la vida (Bisguerra Alzina, 2005).El modelo Educativo de la UNAN Managua hace referencia que "el proceso de aprendizaje se centra en él y la estudiante La formación profesional está íntimamente vinculada a un crecimiento personal que garantice el pensamiento autónomo y critico...", (Delors, 2005). En el marco educativo, la educación emocional se centra en provocar la necesidad de crear sentimientos para el propio bienestar y el de los otros. Por ejemplo, al generar alegría, sentirla y transmitirla al alumnado se estará educando la inteligencia emocional y, por tanto, para la vida. Es decir, la escuela emocional es la que funciona no sólo a través de lo que dicen o hacen directamente los padres o los docentes, sino también en los modelos que ofrecen a la hora de manejar sus propios sentimientos y emociones. Consecuentemente, para compartir los sentimientos de otra persona (empatía) primero se deben conocer los propios, pues cuanto más se dominan nuestros sentimientos, mejor se pueden comprender los de los otros (Ruíz Palomares, 2014).

Como se ha planteado la educación emocional facilita la comprensión del sentimiento propio y el de los demás, esto confirma la necesidad de formación no solamente en el área académica sino en la expresión y manejo de sentimientos y emociones, siendo este un valor agregado en la vida personal y profesional del estudiantado como futuros profesionales. Tiene como objetivo el desarrollo de competencias Emocionales entendiéndolas como el conjunto de conocimientos, capacidades, habilidades y actitudes necesarias para tomar conciencia, comprender expresar y regular de 
forma apropiada los fenómenos emocionales. Dentro de las competencias emocionales están la conciencia y regulación emocional, autonomía emocional, competencias sociales, habilidades de vida y bienestar (Bisguerra Alzina, 2011).

\section{Inteligencia Emocional (IE)}

El modelo de habilidad de Mayer y Salovey 1997 considera que la IE se conceptualiza a través de cuatro habilidades básicas que son:

"La habilidad para percibir valorar y expresar emociones con exactitud, la habilidad para acceder y/o generar sentimientos que faciliten el pensamiento; la habilidad para comprender emociones y el conocimiento emocional y la habilidad para regular emociones removiendo un crecimiento emocional e intelectual (Mayer \& Salovey, 1997) citado en (Berrocal \& Extremara Pacheco, 2005).

\section{La percepción Emocional}

Es la habilidad para identificar y reconocer tanto los propios sentimientos como los de aquello que te rodean. Implica prestar atención y descodificar con precisión las señales emocionales de la expresión facial, movimientos corporales y tono de voz (Berrocal \& Extremara Pacheco, 2005). Esto significa que los estudiantes en el aula tienen la habilidad de reconocer expresiones emocionales tanto de sus compañeros como del maestro regulando así sus acciones ante determinadas emociones.

\section{La facilitación o asimilación emocional}

Implica tener en cuenta los sentimientos cuando razonamos o solucionamos problemas. Esta habilidad se centra en como las emociones afectan al sistema cognitivo y como nuestros estados afectivos ayudan a la toma de decisiones. También ayudan a priorizar nuestros procesos cognitivos básicos focalizando nuestra atención en lo que es realmente importante.
(Berrocal \& Extremara Pacheco, 2005). Los estudiantes necesitan desarrollar habilidades que les permitan procesar la información emocional, con respuestas más efectivas que les muestre la posibilidad de pensar de forma más concentrada e inteligente en su vida emocional.

\section{La comprensión emocional}

Implica la habilidad para desglosar el amplio y complejo repertorio de señales emocionales, etiquetar las emociones y reconocer en que categorías se agrupan los sentimientos. Además, implica una actividad tanto anticipadora como retrospectiva para reconocer las causas generadoras del estado anímico y las futuras consecuencias de nuestras acciones.

\section{La regulación emocional}

Es la habilidad más compleja de la IE, incluye la capacidad para estar abierto a los sentimientos tanto positivos como negativos y reflexionar sobre los mismos para descartar o aprovechar la información que los acompaña en función de su utilidad. Además, incluye la habilidad para regular las emociones propias y ajenas moderando las emociones negativas e intensificando las positivas. (Berrocal \& Extremara Pacheco, 2005).

De acuerdo a lo planteado, el desarrollo de cada una de estas habilidades facilitaría el buen desarrollo tanto del cociente intelectual como del cociente emocional, formando al estudiantado como seres capaces de resolver problemas, siendo asertivos en la toma de decisiones, gestores y sus emociones, mostrando autoestima adecuada, desarrollando habilidades sociales a través del trabajo en equipo.

\section{Las Competencias Emocionales}

Del constructo de inteligencia emocional deriva el desarrollo de competencias emocionales. La competencia es el conjunto de conocimientos, 
capacidades, habilidades y actitudes necesarias para realizar actividades diversas con un cierto nivel de calidad y eficacia. En el concepto de competencia se integra el saber, saber hacer y saber ser. El dominio de una competencia permite producir un número infinito de acciones no programadas (Bisguerra Alzina, 2003, pág. 15).

En la actualidad las competencias emocionales en el campo de la empleabilidad son de gran importancia; el mundo laboral reconoce que la productividad y efectividad depende de una fuerza laboral emocionalmente competente, no basta con tener un buen rendimiento académico tomando únicamente como parámetros las ciencias duras como se plantea desde el enfoque positivista que aparece con Augusto Comte. En su afán por superar la metafísica en los estudios sociales y establecer demarcaciones entre lo científico y lo no científico, se instauró un reduccionismo que preferencia lo estadístico y la información empírica en detrimento de la actividad científico teórica; afirmando esta perspectiva con la trasferencia de los métodos de las ciencias naturales al estudio de la sociedad, precisamente al escenario en el que habitan la sensibilidad, las emociones, la afectividad y muchos otros sentimientos contenidos en la vida humana y donde estos métodos pueden ser ineficientes. Por su parte el positivismo instituyó una visión del método que olvida parte del objeto real de la ciencia al absolutizar la dimensión cuantitativa, ofrece conocimientos parcelados, que no tienen en cuenta que los escenarios sociales y naturales se caracterizan por la multiplicidad de dimensiones y con ello, impide asumir una comprensión de sistema (González \& Hernández Alegría).

La posición reduccionista de Comte no responde a las exigencias del mundo globalizado por cuanto el rendimiento académico durante la formación profesional, no deja de ser importante; sin embargo desarrollar capacidad de autogestión, a través de una autonomía personal en la que él o la estudiante sea capaz de buscar ayuda y recursos, con autoestima y actitud positiva ante la vida, siendo optimista, asumiendo los retos académicos y profesionales y de cualquier ámbito de la vida diaria, poniendo en práctica sus habilidades sociales, asertividad y automotivación son variables subjetivas que le darían un agregado más que los conduce al éxito para integrarse y dar respuesta a las demandas de su vida personal, académica, profesional y social.

Las competencias emocionales son habilidades aprendidas y el hecho de poseer una buena conciencia social o de ser hábil en la gestión de las relaciones no garantiza el dominio del aprendizaje adicional requerido para relacionarse diestramente o solucionar un conflicto. Dicho de otro modo las capacidades subyacentes a la IE son una condición necesaria, aunque no suficiente para evidenciar una determinada competencia. (Goleman, 1996).

\section{MÉTODO}

El presente artículo se presenta bajo el paradigma cualitativo de la investigación siendo este el que busca comprender e interpretar la realidad a través del método de análisis documental y la técnica de revisión documental, para ello se ha realizado revisión de literatura e investigaciones desde un sentido crítico y analítico en relación a trabajos existentes sobre las competencias emocionales y el rendimiento académico. A partir de la descripción y explicación de trabajos realizados, se generaran nuevas ideas que darán pautas para ampliar el conocimiento sobre este tema y definir nuevos caminos para construir una estrategia Psicoeducativa que oriente a la mejora de la calidad del rendimiento académico en los y las estudiantes universitarios.

\section{RESULTADOS DEL ANÁLISIS}

Existen trabajos realizados sobre la inteligencia Emocional; tanto en jóvenes estudiantes como a nivel de docentes. En estudios sobre impacto de la Inteligencia Emocional Percibida, Actitudes Sociales 
y Expectativas del Profesor en el Rendimiento Académico (Morales Jiménez \& Lòpez Zafra, 2013), se realizó con el objetivo de comprobar el papel de la inteligencia emocional percibida y las competencias sociales tienen en el rendimiento académico. Además de analizar el papel de las expectativas de los profesores en el rendimiento académico, el estudio refleja que la conducta social influye decisivamente en el rendimiento académico y analiza la influencia de otras variables como la inteligencia emocional y las expectativas del profesor. Las autoras muestran que los estudiantes que presentan actitudes pro sociales, es decir, que muestran en el aula un comportamiento más sensible, empático y de colaboración (y, en definitiva, que se muestran más competentes socialmente) obtuvieron un rendimiento académico superior a sus compañeros que habían presentado comportamientos asociales (de apatía y retraimiento) y antisociales (dominantes y agresivos)

En dicho estudio realizado con estudiantes de secundaria con edades entre 11 y 16 años, en este trabajo se evaluó la inteligencia Emocional utilizando la versión española del Trait Meta-MoodScale (TMMS24) que proporciona las percepciones de los sujetos sobre su inteligencia emocional y por tanto, reflejan el Índice de Inteligencia Emocional Percibida (IEP). Para la evaluación de las actitudes sociales se empleó el cuestionario de actitudes y Estrategias Cognitivo Sociales es un instrumento de valoración objetiva (que se puntúa en una escala tipo Likert de 7 puntos) cuya finalidad es ofrecer una visión de la competencia social de los adolescentes; es decir, de aquellas variables que más facilitan o más dificultan su adaptación social al medio en que viven. Para la evaluación de las expectativas del profesor, en una escala tipo Likert de tres puntos, sus expectativas sobre dos indicadores: el nivel de adaptación general que alcanzaría el alumno y su rendimiento académico esperado.

Estos instrumentos mostraron un índice de consistencia interna de un alpha de Cron Bach poco confiable para cada componente. En este estudio los resultados obtenidos han mostrado la relación existente entre la conducta pro social y el rendimiento académico Los estudiantes que presentan actitudes pro sociales, es decir, que muestran en el aula un comportamiento más sensible, empático y de colaboración ( $\mathrm{y}$, en definitiva, que se muestran más competentes socialmente) obtuvieron un rendimiento académico superior a sus compañeros que habían presentado comportamientos asociales (de apatía y retraimiento) y antisociales (dominantes y agresivos).En este estudio no se encontraron relaciones estadísticamente significativas entre la inteligencia emocional y el rendimiento académico, Los resultados obtenidos en esta muestra de estudiantes, han puesto de manifiesto que la inteligencia emocional y el rendimiento académico no se relacionan directamente, ya que los resultados dependen del instrumento empleado y varían en función del análisis estadístico o la metodología empleada (Morales Jiménez \& Lòpez Zafra, 2013).

De acuerdo a este estudio se puede inferir que quedan vacíos alrededor de este tema y que existen aspectos que hace falta abordar sobre las competencias emocionales y el rendimiento académico en estudiantes universitarios, lo que significa que es necesario realizar estudios más profundos, que no lleguen únicamente a describir la relación de factores, sino a elaborar propuestas basadas en el desarrollo de competencia emociónales y cognitivas con base de la neurociencia, que permitan un mejor desempeño académico en los estudiantes.

En investigaciones realizadas por Peña (2007) sobre el estado de inteligencia emocional en elámbito educativo, se exponen los estudios acerca de la naturaleza de la IE y de sus instrumentos de medida, así como la validez de criterio, respecto a variables relacionadas con el mundo escolar y universitario. Según este estudio basado en el modelo de IE de Mayer y Salovey, la mayoría de los estudios utilizan auto informes los cuales son más adecuados para medir rasgos de personalidad y nivel de auto eficiencia emocional, pero inadecuados para evaluar habilidades cognitivas implicados en el procesamiento de información emocional. Los 
aportes de los investigadores en relación al tema de competencias emocionales, los métodos que han sido utilizados su para evaluación miden el desarrollo de las habilidades emocionales, pero no dicen si esas habilidades se ponen en práctica en la vida diaria. Los auto informes si destacan este aspecto, ya que estos informan del grado de autoeficacia emocional de un sujeto.

Las pruebas de habilidad de la IE nos revelan si un alumno tiene desarrolladas o no estas habilidades, pero no nos dice nada sobre si ese mismo alumno las pone en práctica en su vida diaria, aspecto que sí es destacado en los auto informes ya que éstos nos informan del grado de autoeficacia emocional de un sujeto. Por otro lado, el MSCEIT se ha revelado como la prueba más adecuada hasta el momento para medir las habilidades cognitivas de la IE. .En el ámbito internacional se ha recomendado una tercera vía para medir la inteligencia emocional que es la 3600 o feedback. Es una de las menos utilizadas, pero se está revelando como un recurso adecuado en el proceso de desarrollo y evaluación de los programas de educación emocional (Pena Garrido \& Repetto Talavera, 2008). La prueba permite comparar las valoraciones de tres categorías de diferentes informantes. El propio sujeto, el profesorado y las autoevaluaciones de un estudiante con las del profesorado.

En el trabajo presentado por Costa y Tabernero de la Universidad de Córdoba (España), sobre el rendimiento académico y auto concepto en estudiantes de educación secundaria obligatoria según el género en el 2012, en el presente estudio, los resultados parecen apuntar que el rendimiento académico de los alumnos está correlacionado con el auto concepto académico. Este auto concepto está fuertemente relacionado con el auto concepto familiar, lo que parece justificar la importancia de la familia en el éxito académico del estudiante, y el auto concepto físico. Otra variable que también parece estar correlacionada negativamente con el rendimiento académico es la edad del estudiante. Lo que sugiere la importancia de crear programas de intervención en los que se fomente el auto concepto de los alumnos para mejorar su rendimiento Académico (Costa \& Tabernero, 2012)

Con el presente artículo se pretende, investigar de forma más profunda sobre el desarrollo de las competencias emocionales y el rendimiento académico desde una perspectiva integral, tomando en cuenta tanto elementos cuantificables, medibles, como otras variables subjetivas que forman parte del comportamiento de los seres humanos.

\section{CONCLUSIONES}

Los aportes de las investigaciones realizadas en torno al tema competencias Emocionales y rendimiento académico, encontrando muchos estudios sobre la inteligencia emocional, evidencian su importancia en el ámbito educativo. Las investigaciones se han centrado en un grupo de la población (niños y adolescentes de 13- 17 años) dejando un vacío con grupos de jóvenes, en este caso con estudiantes a nivel universitario. Los estudios hacen énfasis en la necesidad de diseñar, aplicar y evaluar programas centrados en el desarrollo de las habilidades Emocionales que contribuyan a mejorar el rendimiento académico. De igual forma los estudios hacen notar que existe una necesidad de diseñar o validar instrumentos de medición de las competencias Emocionales que sean objetivos y confiables. Dado que en investigaciones realizadas no ha quedado claro si existen diferentes formas de explicar cómo las personas manejan eficazmente las emociones, por lo que se necesita mayor número de investigaciones con varias medidas validadas de inteligencia emocional (IE) como rasgos y como habilidades (Garrido, 2007). Tomando en cuenta la revisión documental realizada y conocer sobre los aportes realizados por investigadores internacionales en relación a estudios sobre el tema en cuestión considero importante para la construcción de la propuesta psicoeducativa realizar un estudio cuali cuantitativo retomando aspectos de las medidas de evaluación de la inteligencia Emocional, el MSCEIT( El Mayer- Salovey- Caruso Emotional Intelillence test) 
adaptación al castellano por (Extremera y Fernández Berrocal 2002) debido a que aunque no existen muchos datos con este instrumento, es una medida de habilidad emocional con validez factorial de constructo $y$ predicción, que podría brindar aportes importantes en el estudio de la calidad del Rendimiento académico y competencias emocionales, a través de una escala de medición tipo Likert.

El desarrollo de las competencias emocionales no solamente es importantes para mejorar la calidad del rendimiento académico, sino que son competencias para un mejor desempeño en la vida diaria, teniendo su base en la educación Emocional. Esto enfatiza la necesidad de construir una propuesta psicoeducativa como una iniciativa que oriente a la mejora del rendimiento académico. La educación Emocional debe ser un proceso permanente, tanto en estudiantes como docentes, esto conduce al desarrollo integral de la personalidad en los y las estudiantes universitarios y universitarias, es una forma de prevención de la salud mental. En el caso del diseño de una estrategia psicoeducativa vista como un enfoque centrándose en el desarrollo de las competencias emocionales de los y las estudiantes universitarios preparándolos para la vida con miras al éxito en el desarrollo profesional. Es importante mencionar que la vida académica de los y las estudiantes no solamente gira alrededor de la capacidad intelectual del estudiante, el cual siempre se ha visto asociado al rendimiento académico; sino en formar individuos felices, sanos mentalmente, con una adecuada inteligencia emocional, que se planteen retos, capaces de resolver problemas y saber negociar situaciones de la vida cotidiana.

\section{REFERENCIAS BIBLIOGRÁFICAS}

Gaeta González, M. L., \& López Gárcia, C. (2013). Competencias emocionales y rendimiento académico en estudiantes universitarios. Revista Electrónica interuniversitaria de formación del profesorado, 16(2), 13-25.

Agulla Cabrera, V. (2005). El concepto de calidad en la educación universitaria: Clave para el logro de la competitividad institucional. Revista iberoamericana de Educación.

Berrocal, P. F., \& Extremara Pacheco, N. (2005). La Inteligencia Emocional y la educación de las emociones desde el Modelo de Mayer y Salovey.

Bisguerra Alzina, R. (2003). Eduación Emocional y Competencias Básicas para la Vida. Revista de Investigación Educativa, 21(1), 7-43. Recuperado el 2016

Bisguerra Alzina, R. (2011). Educación Emocional Propuesta para Educadores y Familias. España: Desclée de Brower.

Costa, S., \& Tabernero, C. (2012). Rendimiento Académico y autoconcepto en estudiantes de Educación Secundaria Obligatoria según el género. Revista Iberoamericana de Psicología y Salud, 3(2), 175-193. Recuperado el Agosto de 2017, de http://www.redalyc.org/articulo

Delors, J. (2005). Informe de la UNESCO de la comisión internacional sobre la educación para el siglo XXI. Paris: UNESCO. Obtenido de http:// www.unesco.org/education/pdf/DELORS_S.PDF

Garbanzo Vargas, G. M. (2007). Factores Asociados al Rendimiento académico en estudiantes Universitarios, una Reflexión desde la Calidad de la Educación Superior Pública. Educación(1). Recuperado el Noviembre de 2016, de http://www. redalyc.org/articulo.oa?id $=44031103$

Goleman, D. (1996). Inteligencia Emocional. (D. G. Raga, \& F. Mora, Trads.) Barcelona, España: Editorial Kairós.

González, A. M., \& Hernández Alegría, A. (s.f.). Positivismo, Dialéctica y Fenomenoñogía: Tres Enfoques Filosóficos del Método Científico y la Investigación Educativa. Actualidades Investigativas en la Educación, 14(3).

Ibarra, M. d., \& Michalus, J. C. (2010). Análisis del Rendimiento Académico Mediante un Modelo Logit. Recuperado el Noviembre de 2016

Linares Ramos, V. (2009). Emoción y Cognición: Implicaciones para el Tratamiento. Revista Scielo. Ministerio de Educación, N. (2008). de País 
CONFITEA VI 2008. informe de País, Managua. Recuperado el 2016, de http://www.uil.unesco.org/ fileadmin/multimedia/uil/confintea/pdf/National Reports/Latin\%20America\%20-\%20Caribbean/ Nicaragua.pdf

Morales Jiménez, M. I., \& Lòpez Zafra, E. (2013). Impacto de la Inteligencia Emocional Percibida, Actitudes Sociales y Expectativas del profesor en el Rendimiento Académico. Electronic Journal of Research in Educational Psychology(29). Recuperado el Viernes de Noviembre de 2016

Nicaragua. (2008). Constitucion Politica de la Republica de Nicaragua. Managua, Nicaragua: Hispamer.

Ruíz Palomares, A. (2014). Metodologías innovadoras para promover las competencias emocionales de los docentes. Journal for Educators, , Vol 5 (2), 129. Obtenido de http://www.ugr.es/ jett/index.php

UNAN Managua. (2011). Modelo Educativo, Normativa y Metodología para la Planificación Curricular 2011. Recuperado el Febrero de 2017, de http://pagines.uab.cat/unan_uab_innovadocencia/ sites/pagines.uab.cat.unan_uab_innovadocencia/ files/Modelo_Educativo19_de_septiembre.pdf

UNAN, Managua. (2017). Informe de Gestión 2015-2016. Managua. Managua: Universitaria. Recuperado el Marzo de 2017, de http://www. unan.edu.ni/index.php/destacados/unan-managuainforme-de-gestion/

Vigotsky, L. (2004). Teoía de las Emociones Estudio Histórico Psicológico. Madrid, España: Ediciones Akal S,A. Recuperado el 2016 